INPLASY

PROTOCOL

To cite: Sun et al. Comparing acupoint catgut embedding and Tuina therapies in simple obesity: a protocol for systematic review and metaanalysis. Inplasy protocol

202220084. doi:

10.37766/inplasy2022.2.0084

Received: 20 February 2022

Published: 20 February 2022

Corresponding author:

Sun Jiabao

444153695@qq.com

Author Affiliation:

Changchun University of

Chinese Medicine.

Support: 20200403124SF.

Review Stage at time of this submission: The review has not yet started.

Conflicts of interest:

None declared.

\section{Comparing acupoint catgut embedding and Tuina therapies in simple obesity: a protocol for systematic review and meta-analysis}

Sun, J1; Wang, G².

Review question / Objective: Meta-analysis of the effectiveness of acupoint catgut embedding on obesity. The type of disease is obesity, the intervention is acupoint catgut embedding, and the research method is a randomized controlled trials.

Condition being studied: WHO standards define adult overweight as having a BMI of $25.0-29.9 \mathrm{~kg} / \mathrm{m}^{2}$ and obesity as having a BMI of $30.0 \mathrm{~kg} / \mathrm{m}^{2}$ or higher.

Information sources: China Clinical Trial Registry; China National Knowlodge Infrastructure; Chinese Biomedical Literature Database; Chinese Scientific and Journal Database; Wan Fang Database; PubMed; Embase; Cochrane Library; Web of Science; All the databases and online registration platforms will be searched from inception to February 1, 2022and there will be no language restrictions.

INPLASY registration number: This protocol was registered with the International Platform of Registered Systematic Review and Meta-Analysis Protocols (INPLASY) on 20 February 2022 and was last updated on 20 February 2022 (registration number INPLASY202220084).

\section{INTRODUCTION}

Review question / Objective: Meta-analysis of the effectiveness of acupoint catgut embedding on obesity. The type of disease is obesity, the intervention is acupoint catgut embedding, and the research method is a randomized controlled trials.
Condition being studied: WHO standards define adult overweight as having a BMI of $25.0-29.9 \mathrm{~kg} / \mathrm{m}^{2}$ and obesity as having a BMI of $30.0 \mathrm{~kg} / \mathrm{m}^{2}$ or higher.

\section{METHODS}

Participant or population: Patients diagnosed with impaired glucose 
regulation were not restricted by age, sex or race.

Intervention: Interventions use acupoint catgut embedding.

Comparator: The control group use Tuina.

Study designs to be included: RCTs.

Eligibility criteria: Only include randomized controlled trials(RCT) published or registered before February 1, 2022, rewiew articles, case reports and other studies that do not meet the requirements will be excluded.

Information sources: China Clinical Trial Registry; China National Knowlodge Infrastructure; Chinese Biomedical Literature Database; Chinese Scientific and Journal Database; Wan Fang Database; PubMed; Embase; Cochrane Library; Web of Science; All the databases and online registration platforms will be searched from inception to February 1, 2022and there will be no language restrictions.

Main outcome(s): The primary outcome measure was $\mathrm{BMI}$, weight, WC, $\mathrm{HC}$.

Quality assessment / Risk of bias analysis: Two reviewers will assess the methodological quality of the included studies with the Cochran collaboration tool. The bias of sequence generation, allocation concealment, blinding of participants personnel and outcome assessment, incomplete outcome data, selective outcome reporting, and other bias will be accessed by the two authors. A third reviewer (BLS) will arbitrate the disagreement which appears in the evaluation process. The risk of bias would be classified as low, high, or unclear. We will use relative risk (RR) for dichotomous data. Mean differences (MD) or the standard mean differences (SMD) for continuous data. Using $95 \%$ confidence intervals $(\mathrm{Cl})$ to show the effect sizes.

Strategy of data synthesis: Statistical heterogeneity of included studies will be assessed with a standard $I^{2}$ test, if $I^{2}<50 \%$, the heterogeneity of the texts may be ignored, the fixed-effect model will be applied. While if $I^{2} \geq 50 \%$, statistical heterogeneity will be regarded as significant, the random-effects model will be used. We will make use of funnel plot to assess the reporting bias with more than 10 studies are included. If the funnel is symmetrical, which indicates there is no publishing bias, otherwise, there is. But if the included studies are less than 10, $P$ value will be used. We will make use of RevMan (version 5.3) software to conduct the meta-analysis. Fix-effect model will be applied with the condition that there is no heterogeneity of the results. Otherwise, we will turn to the random-effects model after the clinical heterogeneity has been taken out. Subgroup analysis will be conducted based on gender, age, hospitalization time, or other conditions of participants, if potential heterogeneity exists in the included studies. We will take advantage of Grading of Recommendations Assessment, Development and Evaluation Reliability Study (GRADE) to assess the quality of evidence. The grades are very low, low, moderate, and high.

Subgroup analysis: Subgroup analysis will be conducted based on gender, age, hospitalization time, or other conditions of participants, if potential heterogeneity exists in the included studies.

Sensitivity analysis: Robustness of the results will be assessed by sensitivity analysis performance which will focus on the processing method of missing data.

Country(ies) involved: China.

Keywords: acupoint catgut embedding, obesity, protocol, systematic review.

Contributions of each author:

Author 1 - Sun Jiabao.

Author 2 - Wang G. 